\title{
La résistance en héritage - Place et rôle de la théorie française dans la construction du projet de la revue suédoise Kris
}

\author{
Luc Lefebvre \\ Université de Stockholm - Université Paris-Sorbonne
}

En 1977 naît Kris, revue ambitieuse animée par un projet pluridisciplinaire présenté comme un triptyque dans son sous-titre : "Kritik, estetik, politik ». Aux commandes des premiers numéros (i.e. pendant les années I977-I978) peut-on observer une structure bicéphale, composée d'une rédaction et d'un redaktionsråd ( " conseil rédactionnel »), qui témoigne du fait que Kris s'est constituée à partir de la réunion de deux groupes. Le premier, autour de Stig Larsson, est véritablement à l'origine du projet : Kris ne commence en effet pas au numéro I mais au numéro 5 et s'appelait, avant d'être rebaptisée, Kod, une petite revue amateure spécifiquement consacrée au cinéma. Le second groupe comprend quant à lui les «nouveaux venus » Horace Engdahl, Arne Melberg et Anders Olsson chargés de l'ouverture vers les autres disciplines, et en particulier la littérature et la philosophie qui très vite occupent la majeure partie des pages de la revue. Ces quelques noms forment le cœur de la "première génération » de Kris sur les débuts de laquelle nous proposons ici de nous pencher plus en détail.

Cette revue est en effet un objet très intéressant pour une étude des transferts culturels dans la mesure où elle a fortement contribué à l'implantation des théories continentales en Suède en sélectionnant, réinvestissant voire resémantisant ces dernières au fil des mutations du champ intellectuel local mais aussi de ses propres changements de cap éditoriaux. Si la théorie postmoderne française nous intéresse en premier lieu dans cet article, nous ne pourrons néanmoins pas faire l'économie d'une étude de sa cohabitation avec de multiples références internationales, et notamment l'Allemagne.

How to cite this book chapter:

Lefebvre, L. 20I 5. La résistance en héritage - Place et rôle de la théorie française dans la construction du projet de la revue suédoise Kris. In: Cedergren, M. et Briens, S. (eds.) Médiations interculturelles entre la France et la Suède. Trajectoires et circulations de I945 à nos jours. Pp. I90-204. Stockholm: Stockholm University Press. DOI: http:// dx.doi.org/ıo.ı6993/bad.o. License: CC-BY 


\section{Les transferts de théories vers le Nord dans les années 1970}

Dans son ouvrage incontournable French Theory, François Cusset décrit de manière très détaillée comment les théories françaises, à travers un processus de filtrage et d'homogénéisation dans l'univers très spécifique des campus américains au cours de la fin des années I960 et au début des années I970, ont été retravaillées, resémantisées et adaptées à leur nouveau contexte d'adoption, leur permettant ainsi de gagner un public nouveau. Des auteurs comme Michel Foucault et Jacques Derrida ont ainsi non seulement rencontré la contre-culture américaine de l'époque, ils ont aussi, sur le long terme, intégré le milieu universitaire qu'ils ont contribué à renouveler, notamment via les nouvelles disciplines des études culturelles et de genre dont les ramifications ont porté jusque dans la sphère politique. Au chapitre I 3 de l'étude, Cusset revient rapidement sur la manière dont ces théories ont navigué dans le reste du monde, souvent après avoir subi l'influence des États-Unis, ainsi que le rôle qu'a joué le dialogue intense des années I970-I980 entre les théories françaises et les penseurs critiques allemands :

c'est bien souvent en plaçant en vis-à-vis, en mettant l'une l'autre en perspective théorie française et théorie critique postfrancfortienne [...], que la communauté universitaire mondiale, de Tokyo à Mexico, cherche à élucider les modalités contemporaines du pouvoir et du capital, et les enjeux de la lutte sociale - et qu'elle se forge les outils d'une "théorie-monde" (Cusset, 2003:32I)

Comme nous allons le voir, le cas de la revue suédoise Kris présente de nombreuses similitudes, stratégiques et théoriques, avec ce que décrit Cusset. La revue a toutefois fait jouer des canaux de transmission qui lui sont propres pour discuter des théories de France et d'Allemagne et se les réapproprier.

De fait ses membres principaux, ainsi que bon nombre de ses contributeurs, ont été au cœur des processus de transferts qui se sont effectués principalement dans le cadre de la NSU, l'Université d'été nordique (Nordiska Sommaruniversitetet en suédois). Cette organisation a joué un grand rôle dans la diffusion des savoirs continentaux en Scandinavie en tant qu'institution parallèle aux circuits académiques traditionnels des pays respectifs et comme lieu alternatif de formation et de coopération universitaire. Dans le numéro 27 (I980) de Nordisk Forum, la revue de la NSU, le Norvégien Arnulf Kolstad revient sur les trente ans d'activités de cet organisme fondé en I950 dans l'idée de promouvoir une recherche interdisciplinaire entre les cinq pays nordiques. 
S'imposant très vite comme une structure qui rassemble davantage des jeunes chercheurs, enseignants et étudiants plutôt que des professeurs, la NSU est marquée par un esprit d'ouverture et une volonté assumée de créer un milieu de recherche alternatif ( « ved institusjonens ikkeetablerte karakter, dens offisielle outsider-status, basert på frivillighet og engasjement hos deltagerne, har den preg av et "off-universitet ${ }^{\mathrm{I}}$ " ", Kolstad, I980:6), formant un réseau de groupes de travail à l'œuvre tout au long de l'année. Selon une étude de 1978 commanditée par la direction de l'Université d'été nordique, on compte la même année près de I 500 participants, parmi lesquels 40\% d'étudiants (ibid.). Il n'est dès lors guère étonnant de voir que la NSU a été un terrain privilégié, dans les années I970, de la réflexion critique sur la place de la science dans la société et de la contestation gauchiste de l'impérialisme, inflexion politique qui ne se dissipe qu'à la fin de la décennie - sans pour autant que les membres ne renoncent à leur volonté de maintenir cette alternative académique. Dans ce même numéro de célébration, on peut trouver les hommages rendus à l'Université d'été nordique par certains des membres de Kris, en l'occurrence Arne Melberg, Horace Engdahl et Stig Larsson.

C'est de fait bel et bien du côté de la NSU qu'il faut chercher les sources théoriques du groupe Kod/Kris. Deux publications de 1974 (soit un an avant le premier numéro de Kod) dans la collection des Skriftserier de l'Université d'été nordique retiennent notamment notre attention car elles sont révélatrices des tendances les plus fortes à l'œuvre dans ce milieu de recherche et qui posent en même temps certains jalons de la future revue. Le premier de ces ouvrages est la traduction en norvégien du texte de I972 d'Oskar Negt et Alexander Kluge, Öffentlichkeit und Erfahrung (trad. Offentlighet og erfaring par Rolf Reitan), texte qui connaît un grand retentissement dans les milieux de gauche universitaire scandinave (il est de fait réédité la même année). Le second tome, intitulé Subjekt og tekst - bidrag til semiotikkens teori [ "Sujet et texte - contributions à une théorie de la sémiotique »] (éd. Niels L. Knudsen, Ole Andkjær Olsen et Erik Svejgaard), présente les dernières réflexions des groupes de travail danois en sémiologie et sémiotique. La préface prend soin de faire retour sur l'implantation de ces champs de recherche au Danemark, rappelant le remplacement progressif du structuralisme dans la tradition allant de Ferdinand de Saussure à Algirdas Greimas par la conjonction entre Louis Althusser et les "sémiologues du groupe Tel Quel » (sic, Knudsen, Olsen \& Svejgaard, I974 : 5) parmi lesquels comptent aussi bien Julia Kristeva que Jacques Derrida - par 
effet de décalage temporel, et peut-être aussi par simplification pédagogique et stratégique, l'hétérogénéité du champ français est mise de côté (l'éloignement entre Derrida et les Telqueliens ayant par exemple eu lieu dès I97I). Le tome est divisé en deux parties : la première, intitulée "Semiotik og psykoanalyse ", est consacrée de moitié à la présentation des concepts de Jacques Lacan tandis que la seconde, "Semiotik og skriftanalyse » [ "Sémiotique et analyse de l'écriture »], s’intéresse plus particulièrement à Derrida et à la déconstruction des textes. Cette école sémiotique danoise ne fait d'ailleurs pas l'unanimité dans la NSU : en I973, les groupes de travail norvégiens publient ainsi leur anthologie Strukturalisme og semiologi : muligheter og begrensninger $i$ en semiologisk forskningspraksis [ Structuralisme et sémiologie : possibilités et limitations d'une pratique de recherches en sémiologie "] (éd. Kjell S. Johannessen et Arild Utaker) contre la dérive " ésotérique » de leurs collègues, préférant un examen critique de la tradition structuraliste d'avant le moment derridien.

Le Danemark fait ainsi office de médiateur entre la France et le Nord au sein de la NSU, où les groupes de travail diffusent leurs résultats, soit en proposant des traductions (de l'allemand au norvégien ici), soit en fournissant des introductions et des commentaires des textes lus en langue d'origine (dans le cas de Lacan et Derrida, en français). Dans cette configuration précise, le Nord fait son propre travail de filtrage, d'homogénéisation et d'appropriation sans passer par le Nouveau continent. Une seconde étape de ce travail a ensuite lieu puisque le processus se poursuit de la NSU, espace en marge des institutions nationales, vers les pays respectifs - c'est bien là que se joue, dans le cas de la Suède, le rôle de Kod/Kris qui effectue ce travail de transmission en fonction de son propre projet.

\section{De Kod à Kris : genèse et refonte}

Le passage de Kod à Kris marque, du point de vue politique, l'abandon de la révolution pour une reconfiguration critique et, du point de vue du projet scientifique et esthétique, un déplacement (ouverture puis recentrement) disciplinaire. Cette évolution nous intéresse car elle entraîne un changement de stratégie du point de vue de la transmission de théories par rapport à la NSU : si les premiers numéros reposent largement sur les références théoriques discutées dans le cadre de l'Université d'été nordique, la revue va peu à peu, forte de cette formation alternative, gagner une certaine autonomie pour s'affirmer sur le terrain 
proprement suédois et ce, notamment, via une entreprise de ce qu'on pourrait appeler « internalisation " de la traduction.

Dans l'éditorial du premier numéro de Kod, Stig Larsson insiste plus particulièrement sur l'ambition scientifique de la revue qui est de proposer une alternative dans le champ des études cinématographiques grâce à une approche combinant sémiotique et marxisme. L'enjeu est bien entendu de se démarquer des concurrents suédois à gauche, surtout des marxistes orthodoxes dont les lectures et méthodes jugées simplistes ont prédominé dans la première moitié de la décennie. L'analyse à la manière de $K o d$, elle, se veut consciente de ses propres conditions d'existence et entend interroger ses déterminations historiques et sociales plutôt que de livrer des lectures traditionnelles prétendant s'abstraire de leur historicité - tout en maintenant une perspective qui se veut révolutionnaire. C'est précisément à la démarcation méthodologique que sert le recours aux concepts sémiotiques franco-italiens, empruntés notamment à Umberto Eco, Roland Barthes et Christian Metz². Larsson et Thomas Näsholm le précisent dans l'article à vocation polémique "Talar revolutionen ? " [ La révolution parle-t-elle ? »] clôturant le premier numéro, la sémiotique permet de dépasser les sempiternelles oppositions aussi bien politiques (culture prolétaire contre bourgeoise) que plus spécifiquement méthodologiques (contenu contre forme, avec insistance naïve sur le « message » ou lectures lukàcsiennes en réalisme et " tendance ») caractéristiques des analyses marxistes, oppositions qui ne s'interrogent pas sur les conditions de diffusion et de communication de la culture et de ces messages.

Se dessine déjà ici l'autre pilier du langage théorique de Kod, allemand cette fois-ci, à savoir l'herméneutique (surtout Gadamer, Kod 2 : II-I2) et le concept de proletarische Öffentlichkeit de Negt et Kluge qui viennent compléter l'ouverture vers la société qu'engage la sémiotique. Cette cohabitation dans la lignée des tendances qu'a la NSU autour de 1974 ne dure cependant guère : le changement est perceptible entre les numéros 3 et 4 dans l'article "Filmforskningens teori » ["Théorie de la recherche filmique »] où Larsson, après avoir livré une synthèse des outils théoriques utilisables pour une analyse marxiste novatrice du cinéma, finit par véritablement désavouer la sémiotique du film qui, selon lui, s'interroge trop peu sur sa propre historicité - par quoi elle est incapable de rendre compte des fonctions qui produisent l'idéologie dans le capitalisme tardif. Ce faisant, Larsson déplace le but de la recherche filmique : prenant de la distance avec Les Cahiers du Cinéma et Cinéthique et leur réflexion critique sur 
la représentation comme idéologie bourgeoise, il tranche en faveur de l'approche communicationnelle d'inspiration allemande et d'une critique de la position et de la pratique de la recherche. Il en vient ainsi à récuser le positivisme méthodologique, le fétichisme du fait, de la technique, le morcellement des connaissances - en bref la neutralisation de ce savoir par l'institution :

Jag tror att den scienticism som märker filmsemiotikens framställningar beror på en felaktig syn på vetenskapligt arbete. Man har genomgående uppfattat vetenskapligt arbete som något positivt i sig; vetenskapen som en bubbla av vakuum i klasskampens rum [...]; den vetenskapliga oskulden smutsas av ideologin i sin praxis ${ }^{3}$. (Larsson, 1976: 19)

L'approche communicationnelle et critique exige, pour Larsson, un retour à l'expérience esthétique en elle-même, par-delà le formalisme et le scientisme caractéristiques de la sémiotique, et un dépassement du fétichisme du concept scientifique afin de parvenir à aussi modifier le contexte de la diffusion du savoir, l'horizon utopique étant de produire ce qu'il appelle un "espace public scientifique » ("vetenskaplig offentlighet ", Larsson, I976:20). Ce changement de perspective s'accompagne d'une forme d'autocritique à l'égard des articles précédents et d'une promesse d'amélioration dans le futur - futur qui portera le nom de Kris.

La nouvelle mouture concrétise de fait ce programme et resitue les termes du débat en accordant une place de choix à l'École de Francfort au cours des premiers numéros (Theodor W. Adorno dans Kris 5, i.e. le premier numéro sous le nouveau nom, et Negt/Kluge dans Kris 7). Le premier éditorial, signé de la main de Larsson, fait écho à cette autocritique : "Kris ska bli ett forum för det som ligger utanför det traditionella universitetsarbetet, det vanemässiga tänkandet och det till synes självklara4 » (Larsson, I977 : 4). La ligne désormais fixée est ainsi celle d'une discussion contre la pratique académique décriée précédemment, ce qui a deux répercussions sur la manière d'approcher le savoir. D'un côté, l'utilisation des théories va avoir une vocation plus propédeutique que précédemment : ainsi, chacune d'entre elles va être présentée, commentée, contextualisée et discutée dans un contexte suédois ; de l'autre, il va s'agir d'introduire directement, par la traduction, des textes nouveaux. Le dernier numéro de Kod présentait déjà une traduction inédite et exclusive de Kluge ( Kommentar till det antagonistiska realimsbegreppet » [ «Commentaire sur le concept antagonique de réalisme »], trad. Natasa Durovicova) mais la revue ne commentait alors 
guère le texte, se contentant d'une mise en garde contre le jargon et la difficulté. Dans le premier numéro de Kris en revanche, les traductions suédoises d'extraits choisis de l'Ästhetische theorie d'Adorno, réalisées par les propres soins de l'équipe, sont accompagnées et éclaircies de trois articles (une introduction et deux commentaires par Olsson et Engdahl).

\section{Une reconfiguration critique}

La reformulation du projet est ainsi fondée sur l'abandon de la sémiotique d'inspiration française au profit de la pensée allemande. La référence à l'Ecole de Francfort ne doit cependant plus se lire dans les mêmes termes que précédemment puisque Negt et Kluge, s'ils sont repris, sont discutés et inscrits dans une perspective historique et intellectuelle, et non plus simplement adoptés comme tels. La référence à Adorno est par contre bien plus déterminante si l'on entend caractériser la stratégie que veut se donner Kris, à savoir une prise de distance contre toutes les positions établies, aussi bien contre la tradition esthétique marxiste orthodoxe (comme le faisait déjà $K o d$ ) que contre la culture de masse et l'idéologie dominante - prise de distance qui joue en même temps dans un mouvement dialectique avec l'appartenance nécessaire au système. Il s'agit de manière générale de lutter aussi bien contre le vent soufflant vers la droite (une coalition de centre-droit est au gouvernement de I976 à I982) que contre les attaques à l'encontre de l'art et de la culture provenant de l'anti-intellectualisme de tous bords (aussi bien de la société de consommation capitaliste que de ces mêmes marxistes orthodoxes que la revue décrie). Le premier article, écrit par Arne Melberg, est ainsi une discussion sur le rapport entre l'art, la critique et la société et sur l'idée de négativité s'appuyant tout d'abord sur Adorno puis, dans une autre perspective, sur Jauss et Habermas (conciliant ainsi théorie de la réception, de l'horizon d'attente et de l'identification', et approche communicationnelle). L'article se termine sur le roman Esthétique de la résistance de Peter Weiss, présenté comme négation de toutes les attentes, refus de la lecture de consommation, production d'une expérience esthétique réfléchie et enfin discussion des utopies révolutionnaires.

C'est que la vision politique de Kris est bien différente de Kod: le ton révolutionnaire des premières années cède la place à une critique tout autre. Certes, la citation inaugurale en deuxième de couverture 
du premier numéro (nouvelle formule) donne une certaine coloration au rebaptême avec cette citation de Marx : «En ny revolution är bara möjlig i samband med en ny kris. Men den är också lika säker som krisen $^{6}$ "; cependant, l'éditorial qui fait suite se garde bien des interprétations faciles, réfléchissant sur les concepts de " pensée critique » ou de "socialisme » qui ont selon Larsson perdu leur sens à force d'avoir été constamment revendiqués. Les nombreuses redéfinitions et relectures que la revue fait par la suite de son propre nom contribuent elles aussi à nuancer et questionner les positions : ainsi, dans l'éditorial de Kris 6, la «crise » est avant tout la crise de la pensée de gauche dans le contexte de crise économique. Larsson y constate la fin de l'ambiance contestataire de la décennie écoulée et de la vision utilitariste de l'art qui y était associée, la disparition des confrontations et des oppositions nettes, le changement de situation et de générations. Le projet ici devient un projet de résistance, une manière de maintenir allumé un certain flambeau de la pensée et de l'art afin que les intellectuels de gauche puissent trouver matière à poursuivre leur activité critique dans un contexte défavorable - reprenant la dialectique intérieur/extérieur inspirée d'Adorno. Dans Kris 7-8, qui revient à Negt, cette crise se précise encore davantage en crise du marxisme divisé en "sectes ", simplifié en dogmes ou encore instrumentalisé par les partis au pouvoir (à l'Ouest ou à l'Est). La polysémie de la " crise " se redéploie sans cesse sous des éclairages nouveaux, comme dans l'article inaugural collectif et morcelé du numéro double I7-I 8 (I98I) où jouent différentes ramifications de sens à partir de la racine étymologique du terme. Le texte en vient même à critiquer le défaitisme de la société et des milieux intellectuels qui cultivent une idéologie complaisante de la crise. Les éditoriaux, quant à eux, se font de moins en moins frontalement politiques et se concentrent de plus en plus sur des questions théoriques spécifiques à la littérature et la philosophie. Ils disparaissent en tant que tels après I98 I - tout comme le sous-titre «kritik, estetik, politik » à partir de novembre I984.

Ce retrait apparent du politique semble être concomitant avec le déplacement (non exclusif, rappelons-le) du centre de gravité de la référence allemande à la référence française. On peut lire dans cette alternance un partage des tâches : si la première sert en quelque sorte d'armature à la posture de "résistance intellectuelle " de la revue Kris dans le contexte politique plus général, la seconde a un rôle plus spécifique et à certains égards plus offensif. 


\section{L'offensive d'une nouvelle génération}

Comme le proclame Larsson dans l'éditorial du premier Kris, la revue n'entend pas être conciliante, bien au contraire :

Alltså: en tidskrift [...] som inte bara tilltvingar sig sympati, utan också attack.

Det finns ingen "herrschaftsfreie Kommunikation", ingen ideal konsensus, i vilken sanningarna till slut segrar. Sanningen är en maktfråga ${ }^{7}$. (Larsson, I977 : 4)

Il ne fait ici pas seulement allusion au contexte sociopolitique général : les rapports de forces dans le champ académique sont aussi en jeu. On se souvient des regrets, déjà formulés dans Kod, au sujet de la pratique scientifique en vigueur dans les universités. Avec Kris, la revue n'est plus uniquement localisée à Umeå et gérée par des « dilettantes » (sic) : elle se professionnalise encore davantage en ayant désormais un pied à l'Université de Stockholm ${ }^{8}$. Ainsi, derrière le discours de la "résistance ", il s'agit aussi pour les membres, pour la plupart doctorants, de véritablement renverser le rapport de force entre les institutions académiques et la formation alternative de la NSU pour faire pénétrer cette dernière dans l'université suédoise ${ }^{9}$. Kris entend de cette manière s'inscrire dans le vide laissé entre les différentes approches de la littérature, refusant aussi bien l'utilitarisme militant que les pratiques jugées inactuelles de la recherche suédoise.

Les premières affinités spécifiquement littéraires ne sont chronologiquement ni l'Allemagne, ni la France, mais les Etats-Unis (Harold Bloom et Norman O. Brown). Le numéro I 5 de I980, consacré au récit et à la fiction, s'ouvre sur un hommage en forme d'épitaphe en français à Roland Barthes, puis présente un parcours d'ensemble éclectique entre les références sur le sujet (par ex. dans l'article de "Notes sur la fiction ", "Anteckningar om fiktionen ", de Carl-Johan Malmberg : Lacan, Adorno, Bettelheim, Barthes, Bachelard, Jauss, Girard, Freud, Serres). La revue s'oriente cependant vers l'expérience littéraire, le rapport du sujet à la lecture et à l'écriture, conciliant une approche phénoménologique du texte avec les problématiques de la communication $^{\mathrm{I}}$. Grâce à un commentaire sur La Violence et le Sacré de Girard dans Kris I7/I 8 (I98I), la revue prend également acte du concept de « post-structuralisme » et du caractère flou de cette appellation qu'Olsson résume comme l'ensemble des réflexions et tentatives d'exploration et de dépassement des limites du structuralisme. 
Dans son article "Teorins pjonärer ", M. Gustavsson (2008) évoque l'importance du numéro I 6 de Kris (1980) comme moment fort de l'introduction du poststructuralisme en Suède. Y sont présentées deux références récurrentes dans les années qui suivront : Blanchot et surtout Derrida. C'est un coup double puisque les auteurs s'introduisent mutellement en suédois ${ }^{\mathrm{II}}$ : le numéro s'ouvre en effet avec le texte inédit La loi du genre, qui comprend un commentaire de La Folie du jour de Blanchot ; il est suivi par trois articles (Olsson, Engdahl, Malmberg, qui ont aussi traduit le texte) introduisant la pensée du philosophe, puis par une traduction du texte de Blanchot («Dagens vanvett»par Carl Gustav Bjurström) et une présentation par Roland Lysell du rôle considérable de cette pensée sur les théories ultérieures. Blanchot est cependant quelque peu éclipsé par Derrida qui va véritablement être le " créneau " de la revue en Suède ${ }^{\mathrm{I2}}$. C'est ainsi à Kris que revient le privilège d'offrir les premières traductions en suédois de Derrida, avec un intervalle de temps assez serré par rapport à la production du texte (La loi du genre a été prononcé en I979 à Strasbourg au colloque de P. Lacoue-Labarthe et J.-L. Nancy sur Le Genre). L'article de présentation qui fait suite, "Derrida : poetiken ", écrit par A. Olsson, expose la critique du logocentrisme et en présente l'importance pour le champ suédois :

Ett av de allvarligaste problemen (och missförstånden) i den samtida svenska litteraturen och kritiken är ju just denna oförmåga att skilja på tal och skrift [...]. Denna litteratur och kritik saknar nästan genomgående skriftkaraktär, den är språkligt och estetiskt omedveten; ett symptom på denna omognad är naturligtvis dess starkt självbiografiska drag, den är ju frampratad i talets skenbara omedelbarhet. [...] Pratets litteratur suddar ut de verkliga avbrotten, och skapar harmoni och ideologiska modeller. Den är idealistisk och falsk. Ingen kan förklara detta på ett tydligare sätt än Jacques Derrida ${ }^{13}$. (Olsson, I980:45)

Le Derrida de Kris est donc un Derrida aux enjeux littéraires, assez différent du Derrida des sémioticiens danois. L'expérience est par la suite réitérée en I98 I dans le numéro I9, où un compte-rendu d'Éperons recroise Blanchot (extraits en traduction de L'Ecriture du désastre, soit un an après la publication française) et le numéro 20-2I consacré à Hegel, où des pages de Glas en français sont reproduites, présentées et traduites en suédois; en 1983 (Kris 25-26), avec une traduction du commentaire de Kafka Devant la loi, ou encore en 1987 (Kris 3I-32) avec la traduction du Shibboleth écrit pour Paul Celan. 
Très intéressants dans une perspective traductologique, ces textes témoignent d'une grande volonté de transparence de la part des auteurs devant les difficultés linguistiques posées par Derrida ; ainsi pour $L a$ Loi du genre, proposé en version bilingue (suédois et français en vis-àvis) avec une notice expliquant les différents sens de genre en VO; de même pour les commentaires de la traduction de Shibboleth par Aris Fioretos et Hans Ruin ${ }^{\mathrm{I}}$.

Ces exemples sont révélateurs de la stratégie offensive de Kris qui joue sur un double tableau au sens où la revue regarde à la fois le passé, souhaitant combler le retard de l'institution suédoise, et le présent, en étant très attentive à l'actualité continentale. De ce point de vue, la médiation anglo-saxonne joue un rôle très secondaire, bien que parfois complémentaire. On le voit notamment dans le numéro I I-I2 (I979) consacré à la sexualité qui se propose de rediscuter les luttes de la décennie, les nouvelles perceptions de l'individu et des corps qui se sont dégagées, ainsi que les dérives de récupération par la société de consommation qui les menacent. Au cours de sa présentation des théories de Lacan, C.-J. Malmberg reconnaît sa dette à l'égard de l'édition commentée de The Language of the Self par Anthony Wilden (I968) qui lui aurait fourni les clés pour comprendre Lacan, et ce bien qu'il ait en personne assisté à son séminaire (Malmberg, I979 : I9) ; l'introduction fait ensuite place à une traduction (par A. Melberg ${ }^{15}$ ) et un compte-rendu de la critique du même Wilden contre le phallocentrisme de Lacan et ses continuations chez Luce Irigaray. La revue prend par ailleurs position dans ce débat : "Till denna tendens ansluter vi oss gärna med detta nummer av KRIS, åtminstone till de varianter som vi representerar med Wilden och Irigaray och i viss mån Foucault ${ }^{16}$ " (Melberg, I979 : 28). Le cas de Derrida est en revanche différent dans la mesure où Kris s'intéresse moins à lire les textes accessibles en anglais (traductions américaines de Of Grammatology en 1976 par G. Spivak et de Writing and difference en 1978 par $\mathrm{A}$. Bass) et de toute manière déjà connus en langue originale via la NSU, qu'à livrer des traductions en suédois des publications inédites dès le début des années I980. Si le Derrida du champ suédois actuel est volontiers considéré comme " américain ", cela ne doit pas masquer les spécificités et la chronologie du transfert qui s'est effectué, à l'origine, en ligne directe : le Derrida de Kris a bien été le premier à avoir été introduit auprès du public suédois, l'influence des études américaines s'est fait sentir par la suite seulement.

La stratégie double contre le milieu académique suédois se déploie aussi dans le numéro consacré à Hegel. De fait, la critique de la tradi- 
tion universitaire s'y exprime de deux manières : tout d'abord en revenant sur la réception suédoise de la théorie hégelienne depuis le $\mathrm{XIX}^{\mathrm{e}}$ siècle et les obstacles qui ont en ont bloqué l'importation depuis lors, la revue se propose de combler un retard criant de la philosophie et de l'histoire des idées suédoises ; ensuite, bien entendu, en tant que cette réactualisation d'Hegel s'impose comme une manière de renforcer l'armature théorique de la revue, i.e. la tradition dialectique allemande et Derrida - car si le numéro s'ouvre, après la mise en contexte suédois, sur la traduction de la préface à la Phénoménologie de l'Esprit, c'est bien sur Glas que se clôt le numéro. Le double regard critique, tourné à la fois contre le passé et le présent de l'institution, se retrouve encore dans le numéro consacré aux Romantiques allemands (I982, 23-24) : en revenant sur les origines de l'historiographie littéraire, Kris attaquant ouvertement la pratique positiviste suédoise ("Friedrich Schlegel och litteraturhistorien ", Engdahl ${ }^{17}$ ). Reste que ces attaques ciblées veulent ne pas oublier une certaine perspective critique générale - le Hegel de Kris est ainsi considéré comme actuel pour toute interrogation sur le rapport de l'individu au collectif, et non pas simplement pour le jubilé de sa mort ou par rapport à l'arrière-plan du dialogue entre philosophie française et philosophie allemande alors très actuel. C'est du reste aussi ce qui se joue avec Derrida, tel qu'Olsson le présente, pour lutter contre les fausses harmonies et les «modèles idéologiques " évoqués ci-dessus.

\section{Conclusion}

Ainsi, bien qu'il passe de plus en plus au second plan, l'horizon politique doit bel et bien rester à l'esprit du lecteur de Kris - esprit que la revue ambitionne sans relâche de maintenir éveillé, toujours en activité malgré l'adversité régnante et la complexité des années I980. De ce point de vue, la revue constitue un objet de choix pour l'observation des mutations, au fil des numéros, d'une frange du champ littéraire et intellectuel de gauche en Suède après une décennie d'espoirs révolutionnaires. Elle n'en est en outre pas un simple reflet puisqu'elle a activement contribué à ces évolutions. L'offensive dans les murs de l'université suédoise a de fait été couronnée de succès dans la mesure où les départements de littérature ont été, et sont encore aujourd'hui, marqués par cette vague théorique à laquelle la revue a largement participé. Cette dernière ne saurait cependant suffire pour qui veut comprendre la complexité des processus de transferts à elle seule : le cas de Derrida montre bien comment le premier mouvement d'importa- 
tion initié par Kris, qui a puisé directement dans les sources françaises pour nourrir sa discussion critique avec les disciplines philosophiques et littéraires - et en cela a peut-être été plus proche du projet derridien malgré les déplacements inévitables de signification liés au changement de contexte - a été recouvert ultérieurement par les travaux des cultural studies des universités américaines. Néanmoins, parce qu'elle est un agent de médiation par excellence, la revue fournit de nombreuses clés pour approcher les réseaux qui permettent de retracer le voyage de ces objets culturels migrateurs que sont les théories.

\section{Notes}

I. « du fait de son caractère d'institution non-établie, de son statut d'outsider fondé sur le volontariat et l'engagement des participants, elle porte la marque d'une "université off" " (nous traduisons)

2. Aussi bien Eco que Barthes ont été traduits (resp. I971, Den frånvarande struktur, par E. Tenggren et 1966, Litteraturens nollpunkt, par G. et N. A. Bengtsson) chez Bo Cavefors Bokförlag, maison d'édition marquée à gauche dans les années 1960-70. Metz est lu soit en traduction anglaise, soit directement dans la revue Communications.

3. "Je crois que le scienticisme [sic] qui caractérise les représentations de la sémiotique du film reposent sur une vision erronnée du travail scientifique. On a exclusivement compris ce dernier comme quelque chose de positif en soi ; la science comme une bulle de vide dans l'espace de la lutte des classes [...] ; la virginité de la science est, dans la pratique, souillée par l'idéologie. » (nous traduisons)

4. "Kris sera un forum pour tout ce qui se situe en dehors du travail universitaire traditionnel, de la pensée routinière et de ce qui apparaît évident. " (nous traduisons)

5. Melberg s'appuie directement sur les textes allemands, avant tout sur Litteraturgeschichte als Provokation (1970); il fait cependant référence aux traductions de Kurt Aspelin qui a également joué un grand rôle de médiateur (voir à ce sujet Gustavsson, 2008).

6. «Une nouvelle révolution n'est possible qu'en lien avec une nouvelle crise. Mais elle est également aussi certaine que la crise. " (nous traduisons)

7. «Donc : une revue qui ne force pas qu'à la sympathie, mais aussi à l'attaque. Il n'existe pas de "herrschaftsfreie Kommunikation" [de communication libre de tout rapport de domination], aucun consensus dans lequel les vérités finissent par triompher. La vérité est une question de pouvoir. " (nous traduisons). 
8. « Jag tror att det vi kommer att sakna är dilettantismen och charlataneriet. När vi för drygt två år sedan gav ut Kod i Umeå var ingen av oss äldre än nitton. Ingen hade gått på nåt universitet. Teorierna byggdes mest på intuition, på en känsla under fingertopparna. Här, nu, har vi mer motstånd. Tankarna täljs och hamras innan dom presenteras. "(Larsson, I977 : 4. "Je crois que ce qui nous manquera, c'est le dilettantisme et la charlatanerie. Lorsque nous publiions Kod à Umeå il y a tout juste deux ans, aucun d'entre nous n'avait plus de dix-neuf ans. Aucun d'entre nous n'était allé à l'université. Les théories étaient pour la plupart fondées sur l'intuition, sur un sentiment du bout des doigts. Ici, maintenant, nous avons plus de résistance. Les pensées sont taillées au pic et au marteau avant d'être présentées ", nous traduisons)

9. L'antagonisme est déjà perceptible dans le dernier Kod : la présentation de la traduction de Kluge s'attaque aux résistances de l'université suédoise contre la soi-disante "tyskeri » (" allemanderie ») de ceux qui entendent introduire les concepts et problématiques de l'École de Francfort.

ıo. voir l'article de B. Agrell, "Texten som läromästare » [ "Le texte comme maître à penser »], dans ce même numéro, p. 43-5I.

I I. La première traduction de Blanchot en suédois en dehors de Kris date de I990 (Essäer, par Engdahl).

I2. La première traduction nordique de Derrida émane, sans surprise, des sémioticiens danois (Sprog, materialitet, bevidsthed : to essays om metafysikens lukning, Egebak/Larsen, I976).

I 3. «Un des problèmes et des malentendus les plus sérieux dont sont victimes la littérature et la critique suédoises contemporaines est précisément cette incapacité à distinguer oral et écrit [...]. Cette littérature et cette critique manquent presque complètement de caractère écrit, elles n'ont aucune conscience linguistique et esthétique ; un symptôme de cette immaturité est bien entendu leur fort autobiographisme produit dans l'apparente immédiateté de l'oral [...]. La littérature du bavardage efface les véritables ruptures, elle créé une harmonie et des modèles idéologiques. Elle est idéaliste et fausse. Et cela, personne ne saurait l'expliquer avec plus de force que ne l'a fait Jacques Derrida. » (nous traduisons)

I4. A. Fioretos a été élève de Derrida en France ; lui et Ruin sont par la suite les premiers à publier Derrida en suédois au format livresque, à savoir en I 990 avec Schibboleth, texter av Derrida.

I 5. C'est la première traduction de Wilden en suédois ; il faudra attendre I990 pour la suivante (Kommunikationens strategi, trad. de The rules are no game, I987). Les traductions de Lacan en langues nordiques n’ont lieu qu'à partir de la deuxième moitié des années I980.

I6. "C'est à cette tendance que nous nous aimerions nous associer avec ce nu- 
méro de KRIS, ou du moins aux variantes que nous représentons ici par le biais de Wilden, Irigaray et dans une certaine mesure Foucault. » (nous traduisons) I7. Engdahl soutient sa thèse sur le romantisme en 1987 , un an après l'avoir publiée sous forme d'essais chez Bonniers (Den romantiska texten. En essä $i$ nio avsnitt).

\section{Bibliographie des sources citées}

Cusset, F. (2003), French Theory. Paris : La Découverte.

Gustavsson, M. (2008), « Teorins pionjärer : om introduktionen och receptionen av den moderna litteraturteorin i Sverige ". Lychnos, årsbok för idé och lärdomshistoria (2008) : 175-200.

Knudsen, N. \& Olsen, O. \& Svejgaard, E. (éd.) (I974), Subjekt og tekst bidrag til semiotikkens teori. Kongerslev : NSU Skriftserie 5.

Kolstad, A. (I980), " Det tverrvitenskapelige, kritiske studiested i Norden ». Nordisk Forum $27:$ 5-24.

Larsson, S. (1975), « Ledare ». Kod I : 3.

— (I976), «Filmforskningens teori ». Kod $3: 2$ I-23 \& $\operatorname{Kod}_{4}:$ I7-2I.

- (1977), « Ledare ». Kris $5: 4$.

Larsson, S. \& Näsholm, T. (I975), « Talar Revolutionen ? ». Kod I : 28.

Malmberg, C.-J. (I979), « Något om Jacques Lacan ». Kris I I-I 2 : I7-25.

Melberg, A. (I979), « Inledning till en total kybernetisk antropologi - Något om Anthony Wildens negationer ». Kris I I-I 2 : 26-29.

Olsson, A. (I980), « Derrida : poetiken ». Kris I6 : 45-47. 